\title{
Effect of practice and span length on the dual-task coordination executive test
}

\section{R.P. Sylwan, \\ F.M. Rosin and \\ C. Galera}

\author{
Laboratório de Psicologia Experimental Humana, \\ Departamento de Psicologia e Educação, Faculdade de Filosofia, \\ Ciências e Letras de Ribeirão Preto, U niversidade de São Paulo, \\ Ribeirão Preto, SP, Brasil
}

\section{Correspondence \\ F.M. Rosin \\ Laboratório de Psicologia \\ Experimental Humana \\ Departamento de Psicologia e \\ Educação, FFCLRP, USP \\ 14040-901 Ribeirão Preto, SP \\ Brasil \\ Fax: + 55-16-633-6361 \\ E-mail: fabrosin@usp.br}

Part of these data were presented at the Symposium Short- and Long-Term Memory, Porto Alegre, RS, Brasil, November 18-20, 1998, and at the Annual Meeting SAN-SABRO, Puerto Iguazú, Misiones, Argentina, November 30-December 3, 1998.

R.P. Sylwan and F.M. Rosin are recipients of CAPES/SPU fellowships. Publication supported by FAPESP. The first two authors played equal roles in conducting this study.

Received December 2, 1998 Accepted July 28, 1999

\section{Abstract}

The measure " $m u$ ", proposed as an index of the ability to coordinate concurrent box-crossing (BC) and digit-span (DS) tasks in the dual task (DT), should reflect the capacity of the executive component of the working memory system. We investigated the effect of practice in $\mathrm{BC}$ and of a change in the digit span on $m u$ by adding previous practice trials in $\mathrm{BC}$ and diminishing, maintaining or increasing the digit sequence length. The $m u$ behavior was evaluated throughout three trials of the test. Reported strategies in digit tasks were also analyzed. Subjects with diminished span showed the best performance in DT due to a stable performance in DS and BC in the single- and dual-task conditions. These subjects also showed a more stable performance throughout trials. Subjects with diminished span tended to employ effortless strategies, whereas subjects with increased span employed effort-requiring strategies and showed the lowest means of $m u$. Subjects with initial practice trials showed the best performance in $\mathrm{BC}$ and the most differentiated performance between the single- and dual-task conditions in BC. The correlation coefficient between the $m u$ values obtained in the first and second trials was 0.814 for subjects with diminished span and practice trials in BC. It seems that the withinsession practice in $\mathrm{BC}$ and the performance variability in DS affect the reliability of the index $m u$. To control these factors we propose the introduction of previous practice trials in $\mathrm{BC}$ and a modification of the current method to determine the digit sequence length. This proposal should contribute to the development of a more reliable method to evaluate the executive capacity of coordination in the dual-task paradigm.

\section{Introduction}

Executive functions seem to be required to perform two simultaneous tasks involving visuospatial and verbal information. This capacity might indicate the ability to distribute the memory capacity resources and was studied using a dual-task paradigm in the
Key words

- Dual task

- Working memory

- Central executive

- Digit span

- Practice framework of Baddeley and Hitch's working memory model $(1,2)$. Della Sala and colleagues (3) delineated a paper-and-pencil version of the dual-task test by combining the box-crossing (BC) and the digit-span (DS) tasks to contribute to the assessment of patients with Alzheimer's disease or with frontal lesions. Patients with dysexecutive 
syndrome showed an impaired performance in the simultaneous execution of the tasks, whereas they were able to perform the tasks in the single condition at the same level as the normal subjects (3-9).

The dual-task test consists of the execution of the single DS and BC tasks before the combined execution of $\mathrm{DS}$ and $\mathrm{BC}$ in the dual-task condition. The performance in dual task is estimated by $m u$, an index of the ability to coordinate concurrent DS and BC tasks. In a normative study carried out to investigate the statistical behavior of $m u$, Baddeley et al. (6) showed that no significant correlation exists between the index $m u$ and factors like age, gender, educational level and digit span. They also found that the testretest reliability of the obtained $m u$ was lower than what would be required for the clinical use of the test.

In a previous study (10) we extended the investigation of the dual task in healthy subjects. In that study we tested the betweensession training effect on the index $m u$ by the repeated execution of the test and the withinsession practice effect by inverting the order of the tasks. Training affected the singleand the dual-task conditions. The $m u$ values showed stability only when subjects performed the tasks in the direct order of the tasks, but performance was worse in BC under the dual-task condition in the first session when the dual task had been performed before the single task. Thus, withinsession practice could explain some of the better performance in the dual task under the direct order of the tasks. We also found between- and within-subject span length variability affecting $m u$, and a negative correlation between the $m u$ values and the digit span length. We obtained a test-retest reliability coefficient very similar to that reported by Baddeley et al. (6), and concluded that the practice in $\mathrm{BC}$ and a supposed unequal requirement of the subjects' capacities in DS might affect the reliability of the measure $m u$. In the present study we tested the influ- ence of span length and practice in $\mathrm{BC}$ on performance in the dual-task test, by the addition of practice trials in $\mathrm{BC}$ before the dual-task test and by increasing, maintaining or diminishing the digit sequence length. In a previous study (10) we found that the subjects reported effort-requiring strategies for the greater span lengths in DS. Conversely, subjects with lower span lengths tended to report effortless strategies. So, we considered that the diminished-span condition would require less effort-requiring strategies than the increased-span condition. In this study subjects were asked to perform three consecutive trials of the dual-task test to evaluate the within-subject behavior of $m u$.

\section{Material and Methods}

The DS task consists of the repetition of digit sequences presented in a verbal-oral way, which the subject must repeat in the proper order within a 2-min period. Digit sequence length varies according to the subjects' digit span. The BC task consists of traversing with an " $\mathrm{X}$ " a chain of squares linked with arrows to form a path laid out on an A4-size sheet of paper. Subjects are required to fill the squares as rapidly as possible for a period of $2 \mathrm{~min}$. The dual task consists of the simultaneous execution of both tasks within a 2-min period.

To quantify the subjects' performance we used the measures proposed by Baddeley et al. (6). For DS the dependent variables were the percentage of correct repeated digit sequences, for the single-task ( $p s)$ and dualtask $(p d)$ conditions. For $\mathrm{BC}$ the dependent variables were the number of traversed squares, for the single-task ( $t s$ ) and dual-task $(t d)$ conditions. The proportional DS loss $(p m)$ and $\mathrm{BC}$ loss $(p t)$ in the performance in the dual-task condition in relation to the single-task condition was calculated as follows: $p m=p s-p d$ and $p t=(t s-t d) / t s$. The measure $m u$ expressed the relation between the performance of dual and single tasks as a 
percentage, considering the contributions of both tasks to be of equal weight: $m u=[1-$ $(p m+p t) / 2] \times 100$. The performance in the practice trials in $\mathrm{BC}$ was not considered for $m u$ calculation.

Practice in $\mathrm{BC}$ was manipulated by the addition of two practice trials in $\mathrm{BC}$ before the dual-task test. The subjects' capacity requirement in the verbal task was manipulated by increasing, maintaining or diminishing the digit sequence length as a function of the difference between two-span measures. One measure (WS) was considered to be the maximum length repeated once with success based on the WAIS digit test method (11). The other measure, a conservative span (CS), was considered to be the maximum length at which the subject performed all three lists successfully based on the method proposed by Della Sala and colleagues (3). For the diminished-span condition the sequence length obtained with CS was diminished by two digits when WS minus CS was zero, the sequence length obtained with CS was diminished by one digit when the difference between WS and CS was one or two digits, and the sequence length was that obtained with CS when the difference between WS and CS was greater than two. In the maintained-span condition we used the conservative span CS. For the increased-span condition we added two digits to the sequence length obtained with CS when the difference between WS and CS was greater than two, and we added one digit to the CS sequence length when the difference was one or two digits. The same sequence length as CS was used when there was no difference between the CS and WS span length.

Fifty-three healthy volunteers, 37 women and 16 men, participated in this study. Their mean age was 26.0 years \pm SD 6.93 and their mean formal education $16.24 \pm$ SD 3.81 years. The subjects were divided into six groups, three groups with practice trials in $\mathrm{BC}$ and three groups without previous practice trials. As mentioned above, the span for each practice trial condition was increased, maintained or diminished. Eight subjects with increased span, 10 subjects with maintained span and eight subjects with diminished span performed practice trials in BC. Eight subjects with increased span, 11 subjects with maintained span and eight subjects with diminished span did not perform practice trials in BC. The groups were composed according to the subjects' WS and CS lengths. So, the distribution of the span variability was equated across the groups.

Subjects were asked to perform three consecutive trials of the dual-task test to evaluate the within-subject behavior of $m u$. The interval between trials was $5 \mathrm{~min}$. Subjects responded to a questionnaire, which asked them about the strategies employed in the digit sequence recall, the easiest and the hardest task, and the task that received more attention in the dual-task condition.

\section{Results}

The proportion of correct repeated digit sequences, number of traversed squares and $m u$ were analyzed separately. Each of these measures was submitted to ANOVA with four factors: span (increased, maintained and diminished), practice trials (with or without practice), task condition (single and dual) and trials (first, second and third trial).

Table 1 shows the mean number of filled squares in $\mathrm{BC}$ and the proportion of correctly repeated sequences in DS in the single and dual conditions. The mean proportion of correct digit sequences recalled was lower in the dual-task condition than in the singletask condition $[\mathrm{F}(1,47)=8.65, \mathrm{P}=0.005]$, and increased from the first trial to the second, and from the second to the third trial in the single- and dual-task conditions $[\mathrm{F}(2,94)$ $=7.77, \mathrm{P}=0.001]$. Subjects with increased span showed the lowest performance throughout the three trials $[\mathrm{F}(2,47)=13.27$, $\mathrm{P}<0.0001$ ], and their performance was worst in the first trial $[\mathrm{F}(4,94)=3.91, \mathrm{P}=0.006]$. 
The performance in $\mathrm{BC}$ was lower in the dual-task condition than in the single-task condition $[\mathrm{F}(1,47)=31.57, \mathrm{P}<0.0001]$, but it increased in the single- and dual-task conditions across the three trials $[\mathrm{F}(2,94)=78.16$, $\mathrm{P}<0.0001]$. Although not statistically significant $[F(1,47)=3.94, P=0.053]$, subjects with practice trials in $\mathrm{BC}$ showed an in- creased performance throughout the three trials in the single- and dual-task conditions of BC. Analysis of the data demonstrated the interactions practice trials $\mathrm{x}$ task condition $\mathrm{x}$ trials $[\mathrm{F}(2,94)=3.66, \mathrm{P}=0.029]$, and span $\mathrm{x}$ task condition $[\mathrm{F}(2,47)=5.13, \mathrm{P}=0.01]$; these interactions explain the effects of span and practice trials on the loss in $\mathrm{BC}$ under the

Table 1 - Means of filled squares in single- (ts) and dual- (td) box-crossing tasks and proportion of correctly repeated sequences in single (ps) and dual (pd) digit-span tasks.

Standard deviations are given in parentheses.

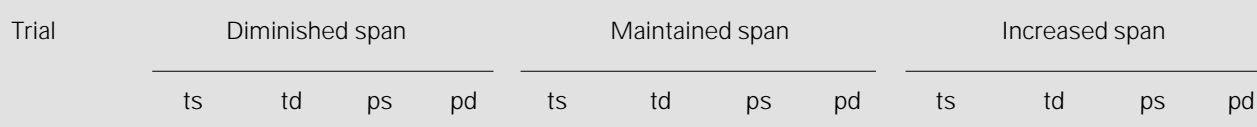

Without practice

$\begin{array}{llllllllllll}167.25 & 163.50 & 1.00 & 0.99 & 165.27 & 156.36 & 0.97 & 0.92 & 175.75 & 162.88 & 0.83 & 0.72\end{array}$ $\begin{array}{lllllllllllll}(15.43) & (16.27) & (0.01) & (0.02) & (27.65) & (27.39) & (0.06) & (0.09) & (22.54) & (38.66) & (0.19) & (0.32)\end{array}$

$\begin{array}{lllllllllllll}3 & 176.13 & 172.75 & 0.99 & 0.98 & 170.91 & 160.09 & 0.95 & 0.95 & 181.88 & 170.13 & 0.87 & 0.83\end{array}$ $\begin{array}{lllllllllllll}(18.40) & (17.60 & (0.02) & (0.03) & (28.51) & (27.94) & (0.06) & (0.09) & (22.80) & (32.57) & (0.24) & (0.17)\end{array}$

With practice

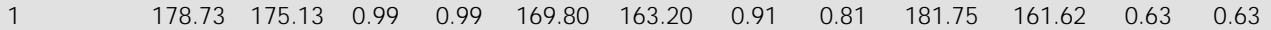
$\begin{array}{lllllllllllll}(20.74) & (25.77) & (0.02) & (0.02) & (31.84) & (29.96) & (0.09) & (0.19) & (10.07) & (15.39) & (0.27) & (0.31)\end{array}$

$\begin{array}{lllllllllllll}2 & 185.75 & 182.75 & 1.00 & 1.00 & 176.10 & 174.00 & 0.88 & 0.85 & 184.75 & 166.50 & 0.78 & 0.64\end{array}$ $\begin{array}{lllllllllllll}(19.37) & (23.86) & (0.00) & (0.00) & (35.18) & (32.60) & (0.13) & (0.24) & (17.23) & (22.06) & (0.25) & (0.36)\end{array}$

$\begin{array}{lllllllllllll}3 & 189.37 & 186.25 & 0.99 & 0.99 & 180.90 & 171.30 & 0.93 & 0.85 & 189.13 & 169.38 & 0.76 & 0.72\end{array}$ $\begin{array}{lllllllllllll}(22.42) & (26.12) & (0.01) & (0.03) & (34.17) & (33.62) & (0.09) & (0.17) & (23.35) & (27.50) & (0.27) & (0.30)\end{array}$

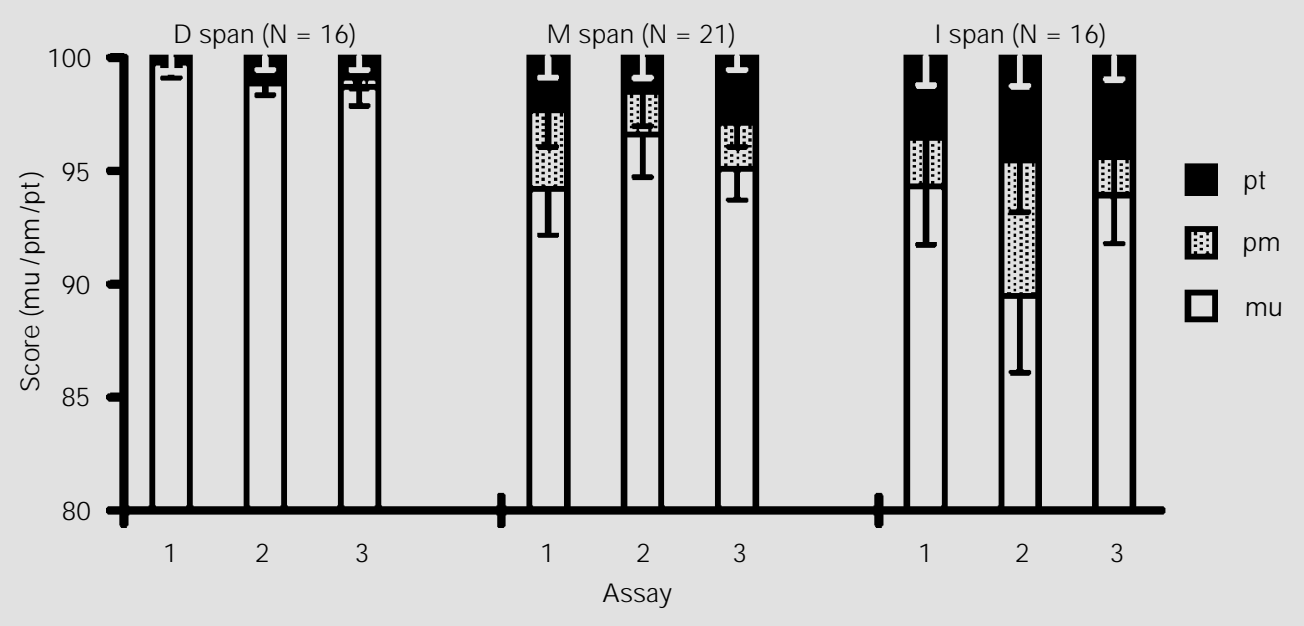

Figure 1 - Scores obtained in the dual task (means \pm SEM). The bars show the contribution to mu by the dual-digit task loss (pm) and the dual-box-crossing loss (pt) throughout trials as a function of span manipulation. D span, M span and I span = Diminished, maintained and increased spans, respectively. 
dual-task condition in relation to the singletask condition. Figure 1 shows that the increased-span group performed BC with the greatest loss under the dual-task condition $[\mathrm{F}(2,47)=4.72, \mathrm{P}=0.014]$. The loss in $\mathrm{BC}$ for the first trial was lower for the subjects without practice trials in $\mathrm{BC}$ and increased across the trials $[\mathrm{F}(2,94)=3.35, \mathrm{P}=0.039]$. So, this result suggests a between-trial practice effect for the group without practice trials in $\mathrm{BC}$ that may have led to an unstable relation between the single- and dual-performance in $\mathrm{BC}$.

The $m u$ values varied according to the span group $[\mathrm{F}(2,47)=3.60, \mathrm{P}=0.035]$. Figure 1 shows that the $m u$ values of the groups with diminished span were the highest for the first session $[\mathrm{F}(4,94)=2.97, \mathrm{P}=$ 0.050]. The group with diminished span showed the most stable $m u$ values across trials. The Pearson correlation coefficient between the $m u$ values obtained in the first and the second trials for the group with the practice trials in $\mathrm{BC}$ and diminished span was $0.814, \mathrm{P}=0.014$. In the present study we did not find a significant correlation between the span length and the $m u$ values for the group with maintained span and without practice trials in $\mathrm{BC}$. This could be due to the reduced size of the sample and to the fact that two subjects with the highest span lengths performed better the DS in the dual-task condition than in the single one, increasing their respective $m u$ values.

Responses to the questionnaire. Subjects reported the strategies employed in DS under the dual-task condition, which were grouped as a function of the required effort. Thus, "just listen and repeat", "to repeat by the sound" and "not using strategies at all" were differentiated from "to imagine the numbers", "to group the numbers", "to hold only the first numbers letting the last ones come by themselves" or "to make associations". Eleven of 14 subjects with diminished span reported effortless strategies, whereas 13 of 15 subjects with increased span reported effort-requiring strategies $\left[\chi^{2}\right.$ $(2, \mathrm{~N}=50)=12.30, \mathrm{P}=0.002]$. The subjects were also asked to report the easiest and hardest tasks (BC, DS or dual task), and which task had received more attention in the dual-task condition (BC and/or DS). The responses were analyzed as a function of span and practice-trial groups and tasks. Boxcrossing was considered the easiest task $\left[\chi^{2}\right.$ $(2, \mathrm{~N}=48)=21.96, \mathrm{P}<0.0001]$. Although without statistical significance, the dual-task condition was considered the hardest task $\left[\chi^{2}(2, N=42)=5.04, P=0.08\right]$. Digit span was the task that received more attention during the dual-task condition $\left[\chi^{2}(2, N=51)\right.$ $=24.27, \mathrm{P}<0.0001]$.

\section{Discussion}

The objective of the present study was to investigate the effect of the practice in $\mathrm{BC}$ and of a change in the digit-span on $m u$, by adding previous practice trials in $\mathrm{BC}$ and diminishing, maintaining or increasing the digit sequence length. The $m u$ behavior was evaluated throughout three trials of the test. We also analyzed the reported strategies in digit tasks. Subjects with increased span showed the lowest performance throughout the three trials and their performance was worst in the first trial, with the greatest loss in $\mathrm{BC}$ under the dual-task condition. The group with diminished span showed the most stable performance across trials and reported mainly effortless strategies, whereas subjects with increased span reported effortrequiring strategies. Thus, the increase in the span requirements seems to lead to the use of effort-requiring strategies in DS, affecting $m u$ stability through a loss in performance in BC.

The loss in $\mathrm{BC}$ for the first trial was lower for the subjects without practice trials in $\mathrm{BC}$ and increased across the trials. So, this result suggests a between-trial practice effect for the group without practice trials in $\mathrm{BC}$ that may have led to an unstable relation between 
the single and dual performance in $\mathrm{BC}$.

The results suggest the possible influence of between-trial practice in $\mathrm{BC}$ and the employed strategies in DS on the reliability of the index $m u$. Although the mechanisms of the interaction between the span length, the practice in $\mathrm{BC}$ and the ability of the dualtask coordination are still unclear, we propose that two things are necessary in order to obtain a higher reliability for the dual-task test: 1 ) a method for the span length determination that reduces the diversity of strategies employed in the digit sequences recall; this can be achieved by diminishing the digit sequence length as a function of the difference between the rather conservative measure of span proposed by Della Sala et al. (3) and the traditional span measure $(11) ; 2$ ) diminishing the within-trial practice by administering previous practice trials in $\mathrm{BC}$.

\section{Acknowledgments}

We are grateful to Prof. A.D. Baddeley and to an anonymous referee for comments on an earlier draft of the paper.

\section{References}

1. Baddeley AD \& Hitch GJ (1974). Working memory. In: Bower GH (Editor), The Psychology of Learning and Motivation. Academic Press, New York, 47-90.

2. Baddeley AD (1986). Working Memory. Oxford University Press, Oxford.

3. Della Sala S, Baddeley A, Papagno C \& Spinnler H (1995). Dual-task paradigm: a means to examine the central executive. Annals of the New York Academy of Sciences, 769: 161-171.

4. Baddeley AD (1996). Exploring the central executive. Quarterly J ournal of Experimental Psychology, 49A: 5-28.

5. Baddeley AD, Bressi S, Della Sala S, Logie R \& Spinnler H (1991). The decline of working memory in Alzheimer's disease. A longitudinal study. Brain, 114: 25212542.

6. Baddeley AD, Della Sala S, Gray C, Papagno C \& Spinnler H (1997). Testing central executive functioning with a pencil-and-paper test. In: Rabbitt P (Editor), Methodology of Frontal and Executive Functions. Chapter 3. Psychology Press, Hove, UK.

7. Baddeley AD, Della Sala S, Papagno C \& Spinnler H (1997). Dual-task performance in dysexecutive and nondysexecutive patients with a frontal lesion. Neuropsychology, 11: 187-194.

8. Baddeley $A D$, Logie R, Bressi S, Della
Sala S \& Spinnler H (1986). Dementia and working memory. Quarterly J ournal of Experimental Psychology, 38A: 603-618.

9. Greene J DW, Hodges J R \& Baddeley AD (1995). Autobiographical memory and executive function in early dementia of Alzheimer type. Neuropsychologia, 33: 16471670.

10. Rosin FM, Sylwan RP \& Galera C (1999). Effect of training on the ability of dual-task coordination. Brazilian J ournal of Medical and Biological Research, 32: 1249-1261.

11. Wechsler D (1995). Teste de Inteligência para Adultos (WAIS). Translated by Álvarez HF. Paidós, Buenos Aires (Original work published in 1955). 